\title{
Relação entre os Sinais de Strain do Ventrículo Direito no Eletrocardiograma e Níveis de Biomarcadores Associados à Gravidade da Pneumonia por COVID-19
}

\author{
Relationship between Right Ventricular Strain Signs in Electrocardiography and Levels of Biomarkers Associated \\ with COVID-19 Pneumonia Severity
}

\author{
Veli Polat, ${ }^{10}$ Evin Bozcali, ${ }^{2}$ Kadriye Kart Yasar, ${ }^{3}$ Hayat Kumbasar Karaosmanoglu, ${ }^{3}$ Ibrahim Faruk Akturk ${ }^{1}$ \\ Departamento de Cardiologia, Bakirkoy Dr. Sadi Konuk Training and Research Hospital, ${ }^{1}$ Istambul - Turquia \\ Departamento de Cardiologia, Prof. Dr. Cemil Tascioglu City Hospital, ${ }^{2}$ Istambul - Turquia \\ Departamento de Doenças Infecciosas e Microbiologia Clínica, Bakirkoy Dr. Sadi Konuk Training and Research Hospital, ${ }^{3}$ Istambul - Turquia
}

\section{Resumo}

Fundamento: A nova doença por coronavírus (COVID-19) pode levar a uma enfermidade grave e causar a morte. Sabe-se que a COVID-19 afeta o sistema cardiovascular. A detecção precoce da progressão para um estágio grave da doença que afeta o sistema cardiovascular pode desempenhar um papel crítico no tratamento da COVID-19.

Objetivos: Explorar a possível relação entre a pneumonia por COVID-19 e os achados de strain do ventrículo direito no eletrocardiograma (ECG).

Métodos: Foi realizado um estudo retrospectivo de 141 pacientes hospitalizados com COVID-19. A correlação de Spearman e as análises de regressão logística foram aplicadas para avaliar as relações entre as manifestações de strain ventricular direito na ECG e os níveis de biomarcadores e outros achados laboratoriais e de imagem do tórax. O nível de significância foi considerado estabelecido como $p<0,05$.

Resultados: Os sinais de ECG de estresse ventricular direito foram significativamente mais frequentes e os níveis de fibrinogênio, PCR e ferritina foram significativamente mais elevados em pacientes com COVID-19 com níveis elevados de hs-cTnl, procalcitonina e dímero-D. A análise univariada mostrou que existem relações significativas entre a presença de pneumonia bilateral, a maioria dos sinais eletrocardiográficos de strain ventricular direito e lesão cardíaca e biomarcadores inflamatórios e trombóticos. A análise multivariada revelou que o supradesnivelamento do segmento ST em V1 e padrão $\mathrm{S}_{1} \mathrm{Q}_{3} \mathrm{~T}_{3}$ são preditores independentes de lesão cardíaca (odds ratio =0,23; IC95\%, 0,06 a 0,90; p=0,035) e níveis elevados de procalcitonina (odds ratio $=0,19 ;$ IC $95 \%, 0,06$ a 0,$62 ; p=0,006$ ), respectivamente.

Conclusão: Os achados do presente estudo sugerem que a dano cardíaco direito é prevalente na COVID-19. Além disso, nosso estudo demonstra o valor clínico do ECG na avaliação e monitoramento de pacientes com pneumonia por COVID-19.

Palavras-chave: COVID-19, Betacoronavirus, Doenças Cardiovasculares, Função Ventricular Direita, Estresse, Eletrocardiografia/ métodos, Biomarcadores, Pneumonia/complicações.

\footnotetext{
Abstract

Background: The novel coronavirus disease (COVID-19) may lead to severe disease that can cause death. COVID-19 is known to affect the cardiovascular system. Early detection of the progression to the severe disease stage that affects the cardiovascular system may play a critical role in the treatment of COVID-19.
}

Objectives: To explore the possible relationship between the COVID-19 pneumonia and right ventricular strain findings on electrocardiography (ECG).

Methods: We conducted a retrospective study of 141 hospitalized patients with COVID-19. Spearman's correlation and logistic regression analyses were applied to assess relationships between ECG manifestations of right ventricular strain and levels of biomarkers and other laboratory and chest imaging findings. The significance level was considered as $<0.05$.

Results: The ECG signs of right ventricular stress were significantly more frequent and the levels of fibrinogen, CRP, and ferritin were significantly higher in COVID-19 patients with elevated levels of hs-cTnl, procalcitonin and D-dimer. The univariate analysis showed there are significant relations between the presence of bilateral pneumonia, most of the ECG signs of right ventricular strain and cardiac injury and inflammatory and thrombotic biomarkers. The multivariate analysis revealed that ST-segment elevation in $V_{1}$ and the $S_{1} Q_{3} T_{3}$ pattern are independent predictors of cardiac damage (odds ratio=0.23; $95 \% \mathrm{Cl}, 0.06$ to $0.90 ; p=0.035$ ) and elevated procalcitonin levels (odds ratio $=0.19 ; 95 \% \mathrm{Cl}, 0.06$ to $0.62 ; p=0.006$ ), respectively.

Correspondência: Veli Polat •

Department of Cardiology, Bakirkoy Dr. Sadi Konuk Training and Research Hospital, Zuhuratbaba Mah, Dr. Tevfik Sağlam Cd No:11, 34147 Bakırköy/Istanbul - Turquia Email: dr.velipolat@gmail.com

Artigo recebido em 29/06/2020, revisado em 20/08/2020, aceito em 14/10/2020

DOI: https://doi.org/10.36660/abc.20200724 
Conclusion: The findings of the present study suggest that right heart damage is prevalent in COVID-19. In addition, our study shows the clinical value of ECG in evaluating and monitoring the patients with COVID-19 pneumonia.

Keywords: COVID-19; Betacoronavirus; Cardiovascular Diseases; Ventricular Function Right; Stress; Eletrocardiography/methods; Biomarkers; Pneumonia/complications.

Full texts in English - http://www.arquivosonline.com.br

\section{Introdução}

A nova pneumonia por coronavírus (COVID-19) de 2019, surgida pela primeira vez em Wuhan, na província chinesa de Hubei, em dezembro de 2019, se espalhou rapidamente pelo mundo. Embora os sintomas respiratórios sejam sinais clínicos primários de COVID-19, vários pacientes também desenvolvem lesão cardiovascular, que é predominantemente detectada como um aumento nos testes de troponina cardíaca de alta sensibilidade. ${ }^{1}$ Além disso, foi demonstrado que a pneumonia por COVID-19 tem uma pior evolução clínica em pacientes com doença cardiovascular. ${ }^{2}$ Nesse contexto, revelar as maneiras pelas quais o novo coronavírus afeta o sistema cardiovascular é crucial para o tratamento desses pacientes de maneira oportuna e eficiente e para a redução de desfechos nefastos, como a morte.

No presente estudo, examinamos as manifestações anormais do eletrocardiograma (ECG) na COVID-19. Especificamente, nosso objetivo foi investigar a possível relação entre a gravidade da pneumonia por COVID-19 e os achados de strain do ventrículo direito no ECG. Além disso, objetivamos explorar possíveis relações entre as anormalidades eletrocardiográficas, dano cardíaco, bem como biomarcadores elevados de inflamação e trombose em pacientes com COVID-19.

\section{Materiais e métodos}

\section{Pacientes do estudo}

Este estudo retrospectivo, de centro único e observacional, foi conduzido no Bakirkoy Dr. Sadi Konuk Training and Research Hospital. Entre 1ํ de abril e 30 de abril de 2020, 141 pacientes consecutivos hospitalizados com diagnóstico de pneumonia por COVID-19, de acordo com as orientações provisórias da Organização Mundial da Saúde, ${ }^{3}$ foram incluídos no presente estudo. O diagnóstico de infecção por COVID-19 também foi confirmado pelo resultado positivo da análise por reação em cadeia da polimerase da transcrição reversa em tempo real de amostras de esfregaço nasal ou faríngeo do paciente. Os ECGs dos pacientes foram obtidos na admissão hospitalar. Todos os pacientes do estudo eram adultos (maiores de 18 anos) e o diagnóstico de embolia pulmonar foi excluído pelos achados de tomografia computadorizada (TC) de tórax com contraste em todos os pacientes.

\section{Coleta de dados}

Os dados demográficos, epidemiológicos, clínicos e radiológicos, bem como as comorbidades e os achados laboratoriais na admissão, foram registrados nos prontuários eletrônicos de cada paciente do estudo. Os dados laboratoriais incluíram a troponina I cardíaca de alta sensibilidade (hs-
cTnl), creatina quinase MB (CK-MB), hemograma completo, proteína- $C$ reativa $(P C R)$, procalcitonina, fibrinogênio, dímero-D, ferritina, testes de função renal e hepática. Todos os pacientes foram acompanhados clinicamente até 25 de maio de 2020. Dois investigadores controlaram todos os dados do estudo.

Níveis elevados de hs-cTnl, que é o sinal de dano cardíaco, foram considerados quando acima do percentil 99 do limite superior de referência. Os níveis de procalcitonina e dímero-D foram considerados elevados quando $\geq 0,5 \mathrm{ng} / \mathrm{mL}$ e $\geq 0,5$ $\mu \mathrm{g} / \mathrm{mL}$, respectivamente. ${ }^{4}$

\section{Avaliação Eletrocardiográfica}

Os ECGs de 12 derivações obtidos no momento da admissão foram interpretados independentemente em termos de sinais de strain do ventrículo direito por dois cardiologistas experientes que desconheciam os dados dos pacientes. Os sinais de ECG foram determinados com base nas manifestações do ECG devido ao estresse ventricular direito secundário à hipertensão pulmonar no embolismo pulmonar. ${ }^{5,6}$ Os seguintes sinais do ECG de strain ventricular direito foram avaliados: (1) taquicardia sinusal (frequência cardíaca $>100$ batimentos/minuto), ${ }^{5}$ (2) bloqueio de ramo direito (BRD) ${ }^{5}$ incompleto ou completo, (3) inversão da onda T nas derivações $\mathrm{V} 1$ a $V 4,{ }^{5}{ }^{5}$ (4) padrão $\mathrm{S}_{1} \mathrm{Q}_{3} \mathrm{~T}_{3}{ }^{5}{ }^{5}(5)$ morfologia $\mathrm{Qr}$ na derivação $\mathrm{V} 1$ (existência de Onda $\mathrm{Q}$ proeminente $\geq 0,2$ milivolts quando a duração do QRS é <120 milissegundos) ${ }^{6}$, (6) elevação do segmento ST $\geq 0,1$ milivolts na derivação V1. ${ }^{6}$

\section{Análise Estatística}

A estatística descritiva foi realizada utilizando-se média \pm desvio padrão (DP) para distribuição normal ou mediana e intervalos interquartis (IIQ) para variáveis contínuas distribuídas assimetricamente e porcentagem para variáveis categóricas. A distribuição das variáveis foi avaliada pelo teste de Kolmogorov-Smirnov. As variáveis contínuas foram comparadas pelo teste $t$ de amostras independentes quando distribuídas normalmente ou pelo teste U de Mann-Whitney quando não distribuídas normalmente. As variáveis categóricas foram comparadas com o teste do qui-quadrado $\left(\mathrm{X}^{2}\right)$ ou o teste exato de Fisher, quando apropriado. As correlações entre os níveis de hs-cTnl, procalcitonina, dímero-D, PCR, fibrinogênio, ferritina e contagem de neutrófilos foram avaliadas pela análise do coeficiente de correlação de Spearman. A análise de regressão logística univariada foi realizada para testar a relação entre as variáveis e os níveis elevados de hs-cTnl, procalcitonina e dímero-D. Além disso, os valores dos odds ratios e intervalos de confiança (IC) de 95\% foram calculados para cada variável. A análise de regressão logística multivariada foi realizada para avaliar os preditores independentes de dano cardíaco (níveis elevados de hs-cTnl), 
níveis elevados de procalcitonina e de dímero-D. As variáveis que foram significativas na análise univariada foram incluídas na análise multivariada. Um valor de $p<0,05$ foi considerado estatisticamente significativo. Todas as análises estatísticas foram realizadas com o software IBM SPSS, versão 26.0.

\section{Resultados}

Foram analisados no presente estudo cento e quarenta e um pacientes consecutivos hospitalizados com pneumonia por COVID-19. A mediana da idade dos pacientes do estudo foi de 64 anos (variação, 26-91 anos) e 84 (59,6\%) eram do sexo masculino. Cinco pacientes morreram durante a hospitalização e 136 pacientes receberam alta. Comorbidades, sinais eletrocardiográficos de strain ventricular direito, achados radiográficos e a evolução clínica de todos os pacientes do estudo são mostrados na Tabela 1 .

\section{Características clínicas e eletrocardiográficas}

A Tabela 2 mostra um resumo da comparação de dados demográficos, sinais de ECG, achados da TC, desfecho clínico e dados laboratoriais, ao categorizar em termos de níveis elevados ou normais de hs-cTnl, procalcitonina e dímero-D em pacientes com pneumonia por COVID-19. Tabagismo, gênero e presença de comorbidades foram semelhantes entre os pacientes com níveis elevados ou normais de hs-cTnl, procalcitonina e dímero-D.
Nos pacientes com níveis elevados de hs-cTnl, procalcitonina e dímero-D, os sinais de ECG de estresse ventricular direito, incluindo taquicardia sinusal, BRD total, inversão da onda $\mathrm{T}$ em $\mathrm{V} 1-\mathrm{V} 4$, padrão $\mathrm{S}_{1} \mathrm{Q}_{3} \mathrm{~T}_{3}$, morfologia $\mathrm{Qr}$ na derivação V1 e supradesnivelamento do segmento ST em V1 foi significativamente mais prevalente em comparação com os pacientes com níveis normais de hs-cTnl, procalcitonina e dímero-D. A proporção de pneumonia bilateral com base nos achados da TC de tórax foi significativamente maior em pacientes com níveis elevados de hs-cTnl, procalcitonina e dímero-D. Pacientes com níveis elevados de hs-cTnl, procalcitonina e dímero-D também apresentaram níveis significativamente mais elevados de fibrinogênio, PCR, ferritina, CK-MB e a razão neutrófilos-leucócitos mais alta, enquanto mostravam contagem de linfócitos significativamente mais baixa (Tabela 2).

A mortalidade hospitalar foi significativamente maior nos pacientes com níveis elevados de hs-cTnl e procalcitonina. $\mathrm{O}$ BRD incompleto foi significativamente frequente nos pacientes que apresentavam apenas dano cardíaco. Pacientes com níveis elevados de procalcitonina apresentaram contagem de neutrófilos significativamente mais alta do que aqueles com níveis normais de procalcitonina. Os pacientes com níveis elevados de procalcitonina e dímero-D eram significativamente mais velhos e a relação linfócitos/leucócitos era significativamente menor (Tabela 2).

Além disso, os pacientes com dano cardíaco apresentaram níveis significativamente mais elevados de dímero-D e

Tabela 1 - Comorbidades, evolução clínica, achados eletrocardiográficos e radiográficos de pacientes com pneumonia por COVID-19

\begin{tabular}{|c|c|}
\hline & Pacientes $(n=141)$ \\
\hline Tabagismo & $21(14,9 \%)$ \\
\hline \multicolumn{2}{|l|}{ Comorbidades } \\
\hline Hipertensão & $44(31,2 \%)$ \\
\hline Diabetes & $36(25,5 \%)$ \\
\hline Doença arterial coronariana & $27(19,1 \%)$ \\
\hline Doença pulmonar obstrutiva crônica & $5(3,5 \%)$ \\
\hline Malignidade & $2(1,4 \%)$ \\
\hline \multicolumn{2}{|l|}{ Sinais do ECG } \\
\hline Taquicardia sinusal & $87(61,7 \%)$ \\
\hline BRD incompleto & $62(44,0 \%)$ \\
\hline BRD Completo & $28(19,9 \%)$ \\
\hline Inversão da onda T in V1 a V4 & $37(26,2 \%)$ \\
\hline Padrão $\mathrm{S}_{1} \mathrm{Q}_{3} \mathrm{~T}_{3}$ & $52(36,9 \%)$ \\
\hline Qr em $V_{1}$ & $35(24,8 \%)$ \\
\hline Supradesnivelamento de ST em $\mathrm{V}_{1}$ & $54(38,3 \%)$ \\
\hline Achados da tomografia computadorizada do tórax & $7(5 \%)$ \\
\hline Pneumonia unilateral & $55(39,0 \%)$ \\
\hline Pneumonia bilateral & $86(61,0 \%)$ \\
\hline Desfecho clínico & $35(24,8 \%)$ \\
\hline Morte hospitalar & $5(3,5 \%)$ \\
\hline
\end{tabular}

ECG: eletrocardiograma; BRD: Bloqueio do ramo direito. 


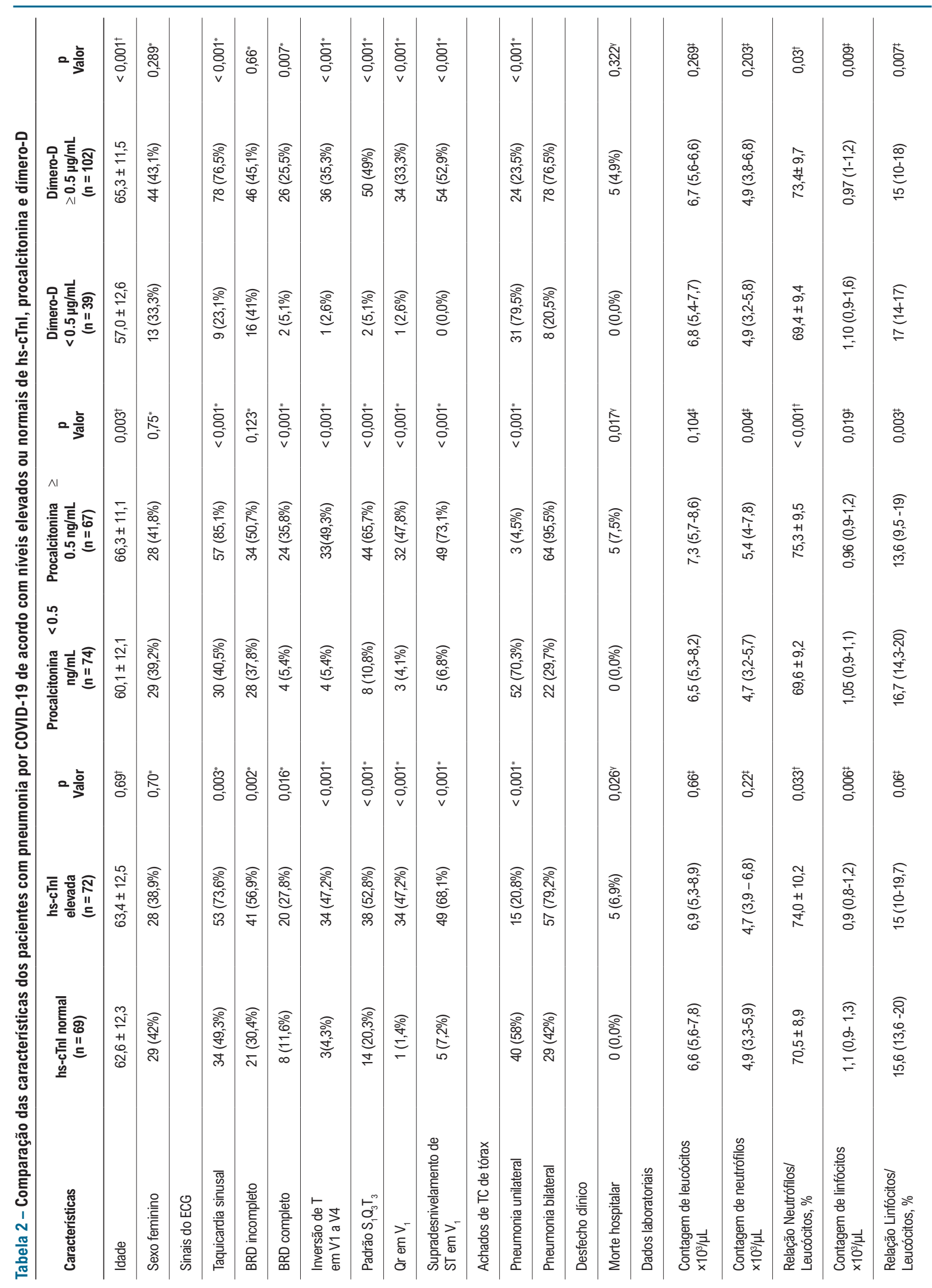




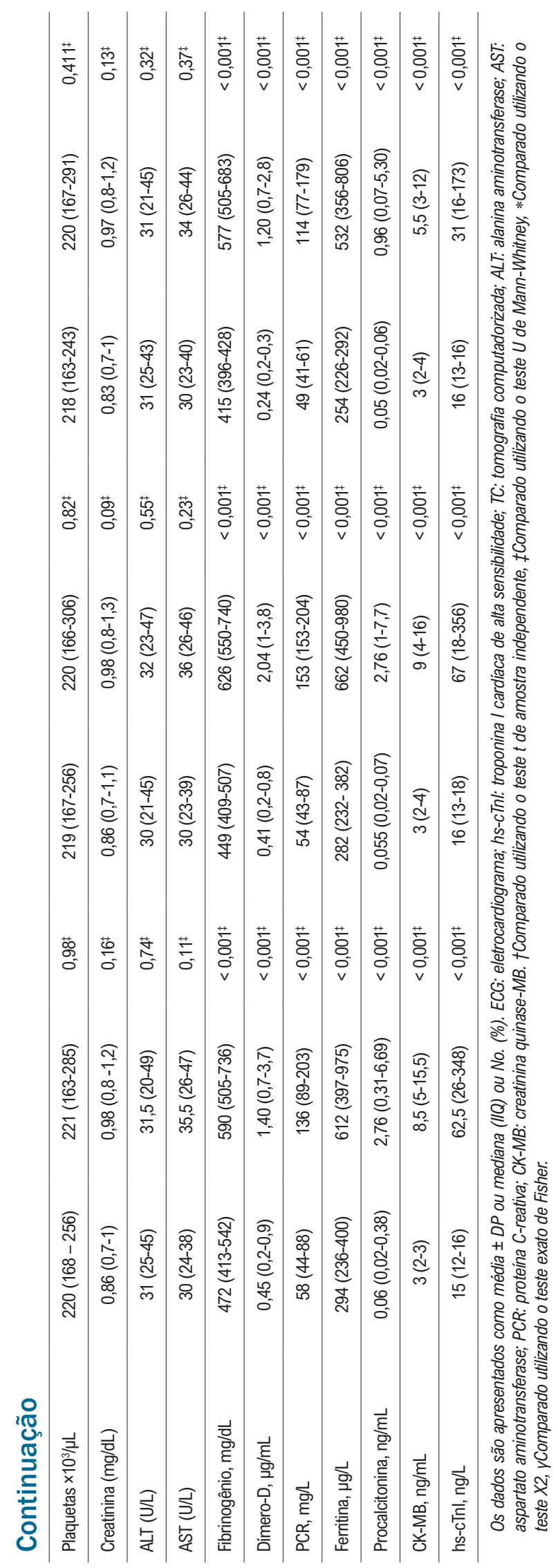

procalcitonina do que aqueles sem danos. Os níveis de dímero-D e hs-cTnl estavam significativamente aumentados em pacientes com níveis elevados de procalcitonina em comparação com pacientes com níveis normais de procalcitonina. Os níveis de procalcitonina e hs-cTnl também estavam significativamente mais elevados em pacientes com níveis elevados de dímero-D em comparação com aqueles com níveis normais de dímero-D (Tabela 2).

\section{Análises de regressão logística univariada e multivariada}

A análise de regressão logística univariada mostrou que taquicardia sinusal, BRD incompleto, BRD completo, inversão da onda $T$ em $V 1-V 4$, padrão $S_{1} Q_{3} T_{3}$, morfologia Qr em V1, supradesnivelamento do segmento ST em V1, presença de pneumonia bilateral, contagem de neutrófilos, PCR, procalcitonina, ferritina, fibrinogênio, dímero-D e CK$M B$ foram significativamente associados ao dano cardíaco em pacientes com pneumonia por COVID-19 (Tabela 3). Entretanto, na análise de regressão logística multivariada, apenas o supradesnivelamento do segmento ST em V1 foi determinado como um preditor independente de dano cardíaco em pacientes com pneumonia por COVID-19 (odds ratio $=0,23$; IC 95\%, 0,06 a 0,90; $\mathrm{p}=0,035)$.

A idade dos pacientes, BRD completo, inversão da onda $\mathrm{T}$ em V1-V4, padrão $\mathrm{S}_{1} \mathrm{Q}_{3} \mathrm{~T}_{3}$, morfologia Qr em V1, supradesnivelamento do segmento ST em V1, presença de pneumonia bilateral, relação linfócitos/leucócitos, contagem de neutrófilos, relação neutrófilos/leucócitos, níveis de PCR, ferritina, fibrinogênio, dímero-D, hs-cTnl, e CK-MB foram significativamente associados com os níveis elevados de procalcitonina na análise de regressão logística univariada. A análise de regressão logística multivariada revelou que o padrão $S_{1} Q_{3} T_{3}$ (odds ratio $=0,19 ;$ IC 95\%, 0,06 a 0,62; $p$ $=0,006$ ), contagem de neutrófilos (odds ratio $=1,35$; IC $95 \%, 1,07$ a 1,$71 ; p=0,011)$ e a presença de pneumonia bilateral (odds ratio =0,15; IC 95\%, 0,03 a 0,84; $p=0,031$ ) foram independentemente associadas aos níveis elevados de procalcitonina (Tabela 4).

Pela análise univariada, a idade dos pacientes, a presença de pneumonia bilateral, a relação linfócito-leucócito, contagens de neutrófilos e linfócitos, níveis de PCR, ferritina, fibrinogênio, procalcitonina, hs-cTnl, CK-MB e presença dos sinais do ECG, incluindo BRD completo, inversão de onda $T$ em V1-V4, padrão $S_{1} Q_{3} T_{3}$, e morfologia Qr em V1 foram significativamente associados com os níveis elevados de dímero-D (Tabela 5). No entanto, entre esses determinantes, apenas o fibrinogênio mostrou ser um preditor independente dos níveis elevados de dímero-D na análise multivariada (odds ratio = 1,05; IC 95\%, 1,03 a 1,07; $\mathrm{p}<0,001$ ).

A análise de correlação de Spearman também revelou que a contagem de neutrófilos, níveis de hs-cTnl, procalcitonina, dímero-D, PCR, fibrinogênio e ferritina estavam significativa e positivamente correlacionados entre si (Tabela 6).

\section{Discussão}

O dano cardíaco detectado pelo aumento de biomarcadores cardíacos na COVID-19 foi associado a um prognóstico fatal. Entretanto, os mecanismos fisiopatológicos subjacentes ao 
Tabela 3 - Determinantes do dano cardíaca em pacientes com COVID-19 por análises de regressão logística univariada e multivariada

\begin{tabular}{|c|c|c|c|c|}
\hline & \multicolumn{2}{|c|}{ Univariada } & \multicolumn{2}{|c|}{ Multivariada } \\
\hline & $\begin{array}{c}\text { OR } \\
(95 \% \mathrm{Cl})\end{array}$ & $\begin{array}{c}p \\
\text { Value }\end{array}$ & $\begin{array}{c}O R \\
(95 \% \mathrm{Cl})\end{array}$ & $\begin{array}{c}\mathbf{p} \\
\text { Valor }\end{array}$ \\
\hline Taquicardia sinusal & $0,35(0,17-0,70)$ & 0,003 & & \\
\hline BRD incompleto & $0,33(0,17-0,66)$ & 0,002 & & \\
\hline BRD completo & $0,34(0,14-0,84)$ & 0,019 & & \\
\hline Inversão T em V1-V4 & $0,05(0,01-0,18)$ & $<0,001$ & & \\
\hline Padrão S1Q3T3 & $0,23(0,11-0,48)$ & $<0,001$ & & \\
\hline Qr em V1 & $0,02(0,00-0,12)$ & $<0,001$ & & \\
\hline Supradesnivelamento do ST em V1 & $0,04(0,01-0,10)$ & $<0,001$ & $0,23(0,06-0,90)$ & 0,035 \\
\hline Presença de pneumonia bilateral & $0,19(0,09-0,40)$ & $<0,001$ & & \\
\hline Contagem de neutrófilos & $1,04(1,00-1,08)$ & 0,036 & & \\
\hline Fibrinogênio, mg/dL & $1,01(1,01-1,01)$ & $<0,001$ & & \\
\hline Dímero-D, $\mu \mathrm{g} / \mathrm{mL}$ & $3,14(1,91-5,17)$ & $<0,001$ & & \\
\hline PCR, mg/L & $1,02(1,01-1,03)$ & $<0,001$ & & \\
\hline Ferritina, $\mu \mathrm{g} / \mathrm{L}$ & $1,01(1,00-1,01)$ & $<0,001$ & & \\
\hline Procalcitonina, ng/mL & $2,03(1,45-2,83)$ & $<0,001$ & & \\
\hline CK-MB, ng/mL & $2,09(1,58-2,76)$ & $<0,001$ & & \\
\hline
\end{tabular}

OR: Odds ratio; IC: intervalo de confiança; BRD: bloqueio de ramo direito; PCR: proteina C-reativa.

Tabela 4 - Determinantes dos níveis elevados de procalcitonina ( $\geq 0,5 \mathrm{ng} / \mathrm{mL})$ em pacientes com COVID-19 por análises de regressão logística univariada e multivariada

\begin{tabular}{|c|c|c|c|c|}
\hline & \multicolumn{2}{|c|}{ Univariada } & \multicolumn{2}{|c|}{ Multivariada } \\
\hline & $\begin{array}{c}\text { OR } \\
(95 \% \mathrm{Cl})\end{array}$ & $\begin{array}{c}p \\
\text { Valor }\end{array}$ & $\begin{array}{c}\text { OR } \\
(95 \% \mathrm{Cl})\end{array}$ & $\begin{array}{c}p \\
\text { Valor }\end{array}$ \\
\hline Idade & $1,04(1,01-1,08)$ & 0,004 & & \\
\hline BRD completo & $0,10(0,03-0,32)$ & $<0,001$ & & \\
\hline Inversão de $T$ em $V_{1}-V_{4}$ & $0,06(0,02-0,18)$ & $<0,001$ & & \\
\hline Padrão $S_{1} Q_{3} T_{3}$ & $0,06(0,03-0,15)$ & $<0,001$ & $0,19(0,06-0,62)$ & 0,006 \\
\hline Qr em V & $0,05(0,01-0,16)$ & $<0,001$ & & \\
\hline Supradesnivelamento de ST em $V_{1}$ & $0,03(0,01-0,08)$ & $<0,001$ & & \\
\hline Presença de pneumonia bilateral & $0,02(0,01-0,07)$ & $<0,001$ & $0,15(0,03-0,84)$ & 0,031 \\
\hline Contagem de neutrófilos & $1,27(1,09-1,48)$ & 0,002 & $1,35(1,07-1,71)$ & 0,011 \\
\hline Relação Neutrófilos/Leucócitos & $1,07(1,03-1,11)$ & 0,001 & & \\
\hline Relação Linfócitos/Leucócitos & $0,93(0,88-0,98)$ & 0,009 & & \\
\hline Fibrinogênio, mg/dL & $1,02(1,01-1,02)$ & $<0,001$ & & \\
\hline Dímero-D, $\mu \mathrm{g} / \mathrm{mL}$ & $8,64(3,67-20,35)$ & $<0,001$ & & \\
\hline PCR, mg/L & $1,03(1,02-1,04)$ & $<0,001$ & & \\
\hline Ferritina, $\mu \mathrm{g} / \mathrm{L}$ & $1,01(1,01-1,01)$ & $<0,001$ & & \\
\hline CK-MB, ng/mL & $1,35(1,18-1,53)$ & $<0,001$ & & \\
\hline hs-cTnl, ng/L & $1,02(1,01-1,02)$ & $<0,001$ & & \\
\hline
\end{tabular}

OR: Odds ratio; IC: intervalo de confiança; BRD: bloqueio de ramo direito; PCR: proteína C-reativa. 
Tabela 5 - Determinantes dos níveis elevados de dímero-D $(\geq 0,5 \mu \mathrm{g} / \mathrm{mL})$ em pacientes com COVID-19 por análises de regressão logística univariada e multivariada

\begin{tabular}{|c|c|c|c|c|}
\hline & \multicolumn{2}{|c|}{ Univariada } & \multicolumn{2}{|c|}{ Multivariada } \\
\hline & $\begin{array}{c}\text { OR } \\
(95 \% \mathrm{Cl})\end{array}$ & $\begin{array}{c}p \\
\text { Valor }\end{array}$ & $\begin{array}{c}\text { OR } \\
(95 \% \mathrm{Cl})\end{array}$ & $\begin{array}{c}p \\
\text { Valor }\end{array}$ \\
\hline Idade & $1,06(1,02-1,10)$ & 0,001 & & \\
\hline BRD completo & $0,16(0,04-0,70)$ & 0,015 & & \\
\hline Inversão de T em V1-V4 & $0,05(0,01-0,37)$ & 0,003 & & \\
\hline Padrão $\mathrm{S}_{1} \mathrm{Q}_{3} \mathrm{~T}_{3}$ & $0,06(0,01-0,25)$ & $<0,001$ & & \\
\hline Qr em V1 & $0,05(0,01-0,40)$ & 0,004 & & \\
\hline Presença de Pneumonia bilateral & $0,08(0,03-0,20)$ & $<0,001$ & & \\
\hline Contagem de neutrófilos & $1,04(1,00-1,09)$ & 0,033 & & \\
\hline Contagem de linfócitos & $0,41(0,17-0,96)$ & 0,041 & & \\
\hline Relação Linfócitos/ Leucócitos & $0,93(0,88-0,98)$ & 0,007 & & \\
\hline Fibrinogênio, mg/dL & $1,04(1,02-1,06)$ & $<0,001$ & $1,05(1,03-1,07)$ & $<0,001$ \\
\hline Procalcitonina, $\mathrm{ng} / \mathrm{mL}$ & $17,73(2,82-111,30)$ & 0,002 & & \\
\hline PCR, mg/L & $1,05(1,03-1,07)$ & $<0,001$ & & \\
\hline Ferritina, $\mu \mathrm{g} / \mathrm{L}$ & $1,01(1,01-1,02)$ & $<0,001$ & & \\
\hline CK-MB, ng/mL & $1,34(1,12-1,61)$ & 0,001 & & \\
\hline hs-cTnl, ng/L & $1,10(1,03-1,18)$ & 0,006 & & \\
\hline
\end{tabular}

OR: Odds ratio; IC: intervalo de confiança; BRD: bloqueio de ramo direito; PCR: proteina C-reativa.

Tabela 6 - Correlações de Spearman dos biomarcadores entre si em pacientes com COVID-19

\begin{tabular}{|c|c|c|c|c|c|c|c|c|c|c|c|c|c|c|}
\hline & \multicolumn{2}{|c|}{ hs-cTnl } & \multicolumn{2}{|c|}{ Procalcitonina } & \multicolumn{2}{|c|}{ Dímero-D } & \multicolumn{2}{|c|}{ PCR } & \multicolumn{2}{|c|}{$\begin{array}{l}\text { Contagem de } \\
\text { neutrófilos }\end{array}$} & \multicolumn{2}{|c|}{ Fibrinogênio } & \multicolumn{2}{|c|}{ Ferritina } \\
\hline & $r$ & $\mathbf{p}$ & $r$ & $\mathbf{p}$ & $r$ & $\mathbf{p}$ & $r$ & $\mathbf{p}$ & $r$ & $\mathbf{p}$ & $r$ & $\mathbf{p}$ & $r$ & $\mathbf{p}$ \\
\hline hs-cTnl & 1 & , & 0,666 & $<0,001$ & 0,633 & $<0,001$ & 0,599 & $<0,001$ & 0,175 & 0,04 & 0,618 & $<0,001$ & 0,625 & $<0,001$ \\
\hline Procalcitonina & 0,666 & $<0,001$ & 1 & , & 0,776 & $<0,001$ & 0,811 & $<0,001$ & 0,213 & 0,01 & 0,748 & $<0,001$ & 0,841 & $<0,001$ \\
\hline Dímero-D & 0,633 & $<0,001$ & 0,776 & $<0,001$ & 1 & , & 0,821 & $<0,001$ & 0,172 & 0,04 & 0,924 & $<0,001$ & 0,842 & $<0,001$ \\
\hline PCR & 0,599 & $<0,001$ & 0,811 & $<0,001$ & 0,821 & $<0,001$ & 1 & , & 0,271 & 0,001 & 0,811 & $<0,001$ & 0,962 & $<0,001$ \\
\hline $\begin{array}{l}\text { Contagem de } \\
\text { neutrófilos }\end{array}$ & 0,175 & 0,04 & 0,213 & 0,01 & 0,172 & 0,04 & 0,271 & 0,001 & 1 & , & 0,174 & 0,04 & 0,222 & 0,008 \\
\hline Fibrinogênio & 0,618 & $<0,001$ & 0,748 & $<0,001$ & 0,924 & $<0,001$ & 0,811 & $<0,001$ & 0,174 & 0,04 & 1 & , & 0,832 & $<0,001$ \\
\hline Ferritina & 0,625 & $<0,001$ & 0,841 & $<0,001$ & 0,842 & $<0,001$ & 0,962 & $<0,001$ & 0,222 & 0,008 & 0,832 & $<0,001$ & 1 & , \\
\hline
\end{tabular}

PCR: proteína C-reativa; hs-cTnl: troponina I cardíaca de alta sensibilidade; $r$ : coeficiente de correlação de Spearman.

dano cardíaco associado à COVID-19 ainda não foram claramente demonstrados. Os possíveis mecanismos que outros estudos sugeriram para explicar o dano miocárdico causado pela COVID-19 são miocardite devido à infecção direta com SARS-CoV-2 ou secundária à resposta inflamatória sistêmica vigorosa induzida pela COVID-19, instabilidade da placa que leva à síndrome coronariana aguda e hipoxemia devido a insuficiência respiratória. ${ }^{7,8}$ Recentemente, trombose microvascular pulmonar foi relatada com dano alveolar difuso na pneumonia por COVID-19.9,10

McGonagle et al. propuseram um modelo de coagulopatia pulmonar intravascular para explicar a lesão miocárdica e a mortalidade cardiovascular na pneumonia por COVID-19. De acordo com este modelo, a COVID-19 induz a coagulopatia pulmonar intravascular difusa através de uma inflamação pulmonar difusa semelhante à síndrome de ativação macrofágica. Tanto a imunotrombose do extenso leito vascular pulmonar quanto a hipoxemia alveolar podem desencadear estresse ventricular direito ao causar hipertensão pulmonar e desempenhar um papel no desfecho fatal. Na pneumonia por COVID-19, níveis elevados de dímero-D podem refletir uma ampla microtrombose pulmonar, enquanto níveis elevados de biomarcadores cardíacos podem indicar strain ventricular direito desencadeado por hipertensão pulmonar. ${ }^{11}$ Os achados do presente estudo apoiam esse modelo. Todos os 
sinais de strain ventricular direito no ECG do estudo, exceto BRD incompleto, foram significativamente mais prevalentes em pacientes com COVID-19 com dano cardíaco e níveis elevados de procalcitonina e dímero-D. Como esperávamos, todos os sinais de strain ventricular direito no ECG, incluindo BRD incompleto, foram significativamente mais comuns em pacientes com COVID-19 com dano cardíaco. Também observamos associação significativa direta entre os níveis de hs-cTnl, procalcitonina e dímero-D, contagem de neutrófilos, níveis de PCR, ferritina e fibrinogênio em pacientes com pneumonia por COVID-19. Esses resultados indicam que a inflamação, trombose e dano cardíaco estão diretamente relacionados entre si em pacientes com COVID-19.

A análise de regressão univariada revelou que quatro dos sete sinais de strain ventricular direito no ECG estavam significativamente associados ao dano cardíaco e níveis elevados de procalcitonina e dímero-D em pacientes com pneumonia por COVID-19. Entretanto, todos os sete sinais do ECG estavam significativamente associados apenas com lesão cardíaca em pacientes com COVID-19. Esses achados sugerem que a possível causa dos níveis elevados de hs-cTnl ou lesão cardíaca em COVID-19 é o estresse ventricular direito que é induzido por hipertensão pulmonar de início súbito devido à trombose vascular pulmonar difusa desencadeada pela pneumonia por COVID-19.

A análise multivariada revelou que entre os sinais do ECG e outras variáveis, apenas o supradesnivelamento do segmento ST em V1 foi um preditor independente de lesão cardíaca em pacientes com pneumonia por COVID-19. Assim, a existência do supradesnivelamento do segmento ST em V1 no ECG à hospitalização pode indicar um mau prognóstico em pacientes com COVID-19, quando consideramos que a lesão cardíaca está associada a prognóstico ruim nesses pacientes. Além disso, a existência de supradesnivelamento do segmento ST em V1 também foi significativamente associada à procalcitonina elevada, de acordo com nossos resultados de análises de regressão. $\mathrm{O}$ padrão $\mathrm{S}_{1} \mathrm{Q}_{3} \mathrm{~T}_{3}$, que é uma manifestação eletrocardiográfica bem conhecida de strain cardíaco direito agudo, particularmente desenvolvido devido à embolia pulmonar maciça, foi identificado como um dos preditores independentes de níveis elevados de procalcitonina em pacientes com pneumonia por COVID-19 em nossas análises de regressão multivariada. Como esperado, os outros dois preditores independentes de níveis elevados de procalcitonina em pacientes com COVID-19 foram a presença de pneumonia bilateral e contagem de neutrófilos. Como esses dois preditores estão relacionados à extensão e à gravidade da doença, sugerimos que o padrão $\mathrm{S}_{1} \mathrm{Q}_{3} \mathrm{~T}_{3}$ também pode refletir a extensão e a gravidade da doença.

Curiosamente, não houve relação entre a idade dos pacientes e os danos cardíacos. Além disso, a análise de regressão univariada demonstrou uma relação positiva entre a idade e níveis elevados de procalcitonina e dímero-D. A análise multivariada indicou o fibrinogênio como o único preditor independente de níveis elevados de dímero-D. No entanto, nenhum dos sinais do strain ventricular direito no ECG foi um preditor independente de níveis elevados de dímero-D. Embora os níveis elevados de dímero-D possam ser o reflexo da trombose intravascular pulmonar difusa na COVID-19, os níveis elevados de dímero-D não são específicos e podem ocorrer devido a muitas outras condições. Portanto, podemos concluir que não encontramos uma relação independente entre os níveis elevados de dímero-D e os sinais no ECG na análise multivariada.

Muito recentemente, Ackermann et al., ${ }^{12}$ publicaram um artigo que investigou os mecanismos patológicos fundamentais da infecção grave por COVID-19, examinando as alterações morfológicas e moleculares dos pulmões obtidas durante a autópsia de pacientes que morreram devido à infecção por COVID-19. Eles relataram que a COVID-19 e a influenza exibem dano alveolar difuso e infiltração de linfócitos perivasculares, embora a COVID-19 seja distinta com suas três características angiocêntricas. Essas características são a trombose vascular extensa com microangiopatia e oclusão de capilares alveolares, a angiogênese vascular via angiogênese intussusceptiva e o dano às células endoteliais possivelmente devido à invasão direta por SARS-CoV-2. ${ }^{12}$ Seus achados apoiam os nossos, mostrando trombose vascular pulmonar generalizada acompanhada por lesão endotelial e angiogênese causada por COVID-19. Portanto, pode-se dizer que o trombo intravascular pulmonar generalizado e a angiogênese induzida por COVID-19 podem levar à hipertensão pulmonar, que pode resultar em um strain súbito do ventrículo direito. Dessa forma, podemos estimar que o estresse do ventrículo direito pode aparecer nos achados do ECG e da ecocardiografia. Em consonância com essa sugestão, mostramos que o estresse ventricular direito pode ser determinado pelo ECG em pacientes com COVID-19 pela primeira vez. Nossos achados também foram corroborados por um estudo recente que demonstrou prevalência de dilatação ventricular direita, também detectada por exame ecocardiográfico, entre os pacientes hospitalizados com infecção por COVID-19. ${ }^{13}$ Entretanto, as relações entre dilatação ventricular direita, dano cardíaco, bem como biomarcadores de inflamação e trombose não foram investigados no estudo acima mencionado.

Nosso estudo tem algumas limitações que precisam ser declaradas. Primeiro, este é um estudo de centro único com um tamanho de amostra pequeno. Em segundo lugar, como é um estudo retrospectivo, investigações laboratoriais específicas, como outros biomarcadores trombóticos e cardíacos ou exames ecocardiográficos, e monitoração de ECG para possível reversão dos sinais do ECG, não estavam disponíveis. Terceiro, como o estudo incluiu apenas os pacientes com COVID-19, os pacientes sem COVID-19 não puderam ser comparados, especialmente em relação aos sinais do ECG.

\section{Conclusões}

Que seja de nosso conhecimento, o presente estudo é o primeiro que mostra as manifestações do strain do ventrículo direito no ECG e sua relação com a inflamação e trombose em pacientes com pneumonia por COVID-19. Além disso, os achados deste estudo revelam que a infecção por COVID-19 afeta o coração, especialmente através do lado direito do coração. Ao fazer isso, nosso estudo eventualmente sugere que o ECG, que é um teste viável, econômico, seguro e rápido, pode ser utilizado tanto na avaliação quanto no 
monitoramento dos pacientes com COVID-19 em termos de dano cardíaco, gravidade da inflamação e talvez prognóstico. Finalmente, nossos achados precisam ser validados através de estudos clínicos prospectivos e maiores.

\section{Contribuição dos autores}

Concepção e desenho da pesquisa e Revisão crítica do manuscrito quanto ao conteúdo intelectual importante: Polat V, Bozcali E; Obtenção de dados: Polat V, Akturk IF; Análise e interpretação dos dados: Polat V, Bozcali E, Yasar KK, Karaosmanoglu HK; Análise estatística e Obtenção de financiamento: Polat V; Redação do manuscrito: Polat V, Bozcali E, Akturk IF.

\section{Potencial conflito de interesse}

Não há conflito com o presente artigo

\section{Referências}

1. Huang C, Wang Y, Li X, Ren L, Zhao J, Hu Y, et al. Clinical features of patients infected with 2019 novel coronavirus in Wuhan, China. Lancet. 2020;395(10223):497-506

2. Li B, Yang J, Zhao F, Zhi L, Wang X, Liu L, et al. Prevalence and impact of cardiovascular metabolic diseases on COVID-19 in China. Clin Res Cardiol. 2020;109(5):531-8.

3. World Health Organization. (WHO). Global surveillance for COVID-19 caused by human infection with COVID-19 virus: interim guidance. [Cited in 2020 March 20] Available from:https://extranet.who.int/iris/restricted/ handle/10665/331506.

4. Guan WJ, Ni ZY, Hu Y, Liang WH, Ou CQ, He JX, et al. Clinical Characteristics of Coronavirus Disease 2019 in China. N Engl J Med. 2020;382(18):1708-20.

5. Daniel KR, Courtney DM, Kline JA. Assessment of cardiac stress from massive pulmonary embolism with 12-lead ECG. Chest. 2001;120:474-481.

6. Kucher N, Walpoth N, Wustmann K, Noveanu M, Gertsch M. QR in V1- an ECG sign associated with right ventricular strain and adverse clinical outcome in pulmonary embolism. Eur Heart J. 2003;24(1):1113-9.

7. Akhmerov A, Marbán E. COVID-19 and the Heart. Circ Res. 2020;126(10):1443-55

\section{Fontes de financiamento}

O presente estudo não teve fontes de financiamento externas.

\section{Vinculação acadêmica}

Não há vinculação deste estudo a programas de pósgraduação.

\section{Aprovação ética e consentimento informado}

Este estudo foi aprovado pelo Comitê de Ética do Bakirkoy Dr. Sadi Konuk Training and Research Hospital sob o número de protocolo 2020/255. Todos os procedimentos envolvidos nesse estudo estão de acordo com a Declaração de Helsinki de 1975, atualizada em 2013. O consentimento informado foi obtido de todos os participantes incluídos no estudo.

8. Guo T, Fan Y, Chen M, Wu X, Zhang L. He T, et al. Cardiovascular implications of fatal outcomes of patients with coronavirus disease 2019 (COVID-19). JAMA Cardiol. 2020;5(7):811-8.

9. Zhang T, Sun LX, Feng RE. Comparison of clinical and pathological features between severe acute respiratory syndrome and coronavirus disease 2019. Zhonghua Jie He He Hu Xi Za Zhi. 2020;43(6):496-502.

10. Yao XH, Li TY, He ZC, Ping YF, Liu HW, Yu SC, et al. A pathological report of three COVID-19 cases by minimally invasive autopsies. Zhonghua Bing Li Xue Za Zhi. 2020;49(5):411-7.

11. McGonagle D, O'Donnell JS, Sharif K, Emery P, Bridgewood C. Immune mechanisms of pulmonary intravascular coagulopathy in COVID-19 pneumonia. Lancet Rheumatol. 2020;2(7):e437-e445.

12. Ackermann M, Verleden SE, Kuehnel M, Haverich A, Welte T, Laenger F, et al. Pulmonary vascular endothelialitis, thrombosis, and angiogenesis in Covid-19. N Engl J Med. 2020;383(2):120-8.

13. Argulian E, Sud K, Vogel B, Bohra C, Garg V, Talebi S, et al. Right ventricular dilation in hospitalized patients with COVID-19 infection. JACC CardiovasC Imaging. 2020;13(11):2459-61. 